Research Article

\title{
Discontinuous Mixed Covolume Methods for Linear Parabolic Integrodifferential Problems
}

\author{
Ailing Zhu \\ School of Mathematical Sciences, Shandong Normal University, Jinan 250014, China \\ Correspondence should be addressed to Ailing Zhu; zhuailing88@126.com
}

Received 4 April 2014; Accepted 17 June 2014; Published 15 July 2014

Academic Editor: Wolfgang Schmidt

Copyright ( 2014 Ailing Zhu. This is an open access article distributed under the Creative Commons Attribution License, which permits unrestricted use, distribution, and reproduction in any medium, provided the original work is properly cited.

The semidiscrete and fully discrete discontinuous mixed covolume schemes for the linear parabolic integrodifferential problems on triangular meshes are proposed. The error analysis of the semidiscrete and fully discrete discontinuous mixed covolume scheme is presented and the optimal order error estimate in discontinuous $H(\mathrm{div})$ and first-order error estimate in $L^{2}$ are obtained with the lowest order Raviart-Thomas mixed element space.

\section{Introduction}

We consider the following linear parabolic integrodifferential problems

$$
\begin{gathered}
q_{t}(\mathbf{x}, t)-\nabla \cdot\left\{A(\mathbf{x}, t) \nabla q(\mathbf{x}, t)+\int_{0}^{t} B(\mathbf{x}, t, \tau) \nabla q(\mathbf{x}, t, \tau) d \tau\right\} \\
=f(\mathbf{x}, t), \quad(\mathbf{x}, t) \in \Omega \times(0, T], \\
q(\mathbf{x}, 0)=q_{0}(\mathbf{x}), \quad \mathbf{x} \in \Omega, \\
q(\mathbf{x}, t)=0, \quad(\mathbf{x}, t) \in \partial \Omega \times(0, T],
\end{gathered}
$$

where $\Omega \in \mathbf{R}^{2}$ is a bounded convex polygonal domain with the boundary $\partial \Omega, \mathbf{x}=(x, y), q$ is an unknown function, $A$ is a symmetric, bounded matrix function, $B$ is a bounded matrix function, $q_{0}(\mathbf{x})$ and $f(\mathbf{x}, t)$ are known functions, and $f(\mathbf{x}, t) \epsilon$ $L^{2}(\Omega)$. Furthermore, we assume that the matrix $M=A^{-1}$ and $M_{t}$ are two bounded matrix functions.

Here and in what follows, we will not write the independent $\mathbf{x}, t$ for any functions unless it is necessary.

For the parabolic integrodifferential problems many numerical methods were proposed, such as the finite element methods in [1], H1-Galerkin mixed finite element methods in [2], finite element approximation with a weakly singular kernel in [3], expanded mixed finite element methods in [4], and expanded mixed covolume method in [5].
Because the discontinuous Galerkin method has the advantages of a high order of accuracy, high parallelizability, localizability, and easy handling of complicated geometries it has been used to solve elliptic problems and convectiondiffusion problems by many researchers; see [6-11]. The discontinuous finite volume method in recent years was used to solve elliptic problems, Stokes problems, and parabolic problems in [12-14]. In [15] the discontinuous mixed covolume methods for elliptic problems were demonstrated by Yang and Jiang. Zhu and Jiang extended the discontinuous mixed covolume methods to parabolic problems in [16]. The goal of this paper is to extend the discontinuous mixed covolume methods in the linear parabolic integrodifferential problems.

The rest of this paper is organized as follows. In Section 2, some notations are introduced and the semidiscrete and the fully discrete discontinuous mixed covolume schemes for the integrodifferential equations (1) are established. In Section 3, the existence and uniqueness for the semidiscrete and the fully discrete discontinuous mixed covolume approximations are proven. We defined a generalized discontinuous mixed covolume elliptic projection in Section 4 . We prove the optimal error estimations in both $H^{1}$ and $L^{2}$ norms of semidiscrete and the fully discrete discontinuous mixed covolume methods in Sections 5 and 6.

Throughout this paper, the letter $C$ denotes a generic positive constant independent of the mesh parameter and may stand for different values at its different appearances. 


\section{Discontinuous Mixed Covolume Formulation}

Let $\mathbf{w}=-A \nabla q$ and $D=B M$, and rewrite (1) as the system of first-order partial differential equations

$$
\begin{gathered}
M \mathbf{w}+\nabla q=0, \quad(\mathbf{x}, t) \in \Omega \times(0, T], \\
q_{t}+\nabla \cdot \mathbf{w}+\int_{0}^{t} \nabla \cdot(D \mathbf{w}) d \tau=f, \quad(\mathbf{x}, t) \in \Omega \times(0, T], \\
q(\mathbf{x}, 0)=q_{0}(\mathbf{x}), \quad \mathbf{x} \in \Omega, \\
q(\mathbf{x}, t)=0, \quad(\mathbf{x}, t) \in \partial \Omega \times(0, T] .
\end{gathered}
$$

We will use the standard definitions for the Sobolev spaces $H^{s}(K)$ and their associated inner products $(\cdot, \cdot)_{s, K}$, norms $\|\cdot\|_{s, K}$ and seminorms $|\cdot|_{s, K}$ in [17]. The space $H^{0}(K)$ coincides with $L^{s}(K)$, in which the norm and the inner product are denoted by $\|\cdot\|_{K}$ and $(\cdot, \cdot)_{K}$, respectively.

Let $\mathscr{T}_{h}=\{K\}$ be a triangulation of the domain $\Omega$. As usual, we assume the triangles $K$ to be shape-regular. For a given triangulation $\mathscr{T}_{h}$, we construct a dual mesh $\mathscr{T}_{h}^{*}$ based upon the primal partition $\mathscr{T}_{h}$. Each triangle in $\mathscr{T}_{h}$ can be divided into three subtriangles by connecting the barycenter $Q$ of the triangle to their corner nodes $A_{i}(i=1,2,3)$. Then we define the dual partition $\mathscr{T}_{h}^{*}$ to be the union of the triangles shown in Figure 1. Let $P_{k}(T)$ consist of all the polynomials functions of degree less than or equal to $k$ defined on $T$. We define the finite-dimensional trial function space for velocity on $\mathscr{T}_{h}$ by

$$
U_{h}:=\left\{\mathbf{u} \in L^{2}(\Omega)^{2}:\left.\mathbf{u}\right|_{K} \in P_{1}(K)^{2}, \forall K \in \mathscr{T}_{h}\right\} .
$$

Define the finite-dimensional test function space $V_{h}$ for velocity associated with the dual partition $\mathscr{T}_{h}^{*}$ as

$$
V_{h}:=\left\{\mathbf{v} \in L^{2}(\Omega)^{2}:\left.\mathbf{v}\right|_{T} \in P_{0}(T)^{2}, \forall T \in \mathscr{T}_{h}^{*}\right\} .
$$

Let $H_{h}$ be the finite-dimensional space for pressure

$$
H_{h}:=\left\{p \in L^{2}(\Omega):\left.p\right|_{K} \in P_{0}(K), \forall K \in \mathscr{T}_{h}\right\} .
$$

Let $\Gamma$ denote the union of the boundary of the triangles $K$ of $\mathscr{T}_{h}$ and $\Gamma^{0}: \Gamma \backslash \partial \Omega$. The traces of functions in $V_{h}$ and $H_{h}$ are double valued on $\Gamma^{0}$. Let $e$ be an interior edge shared by two triangles $K_{1}$ and $K_{2}$ in $T_{h}$. Define the normal vectors $\mathbf{n}_{1}$ and $\mathbf{n}_{2}$ on $e$ pointing exterior to $K_{1}$ and $K_{2}$, respectively. Next, we introduce some traces operators that we will use in our numerical formulation. We define the average $\{\cdot\}$ and jump $[\cdot]$ on $e$ for scalar $q$ and vector $\mathbf{v}$, respectively:

$$
\begin{array}{cc}
\{p\}=\frac{1}{2}\left(\left.p\right|_{\partial K_{1}}+\left.p\right|_{\partial K_{2}}\right), & {[p]=\left.p\right|_{\partial K_{1}} \mathbf{n}_{1}+\left.p\right|_{\partial K_{2}} \mathbf{n}_{2}} \\
\{\mathbf{u}\}=\frac{1}{2}\left(\left.\mathbf{u}\right|_{\partial K_{1}}+\left.\mathbf{u}\right|_{\partial K_{2}}\right), & {[\mathbf{u}]=\left.\mathbf{u}\right|_{\partial K_{1}} \cdot \mathbf{n}_{1}+\left.\mathbf{u}\right|_{\partial K_{2}} \cdot \mathbf{n}_{2} ;}
\end{array}
$$

if $e$ is an edge on the boundary of $\Omega$, we set

$$
\{p\}=p, \quad[\mathbf{u}]=\mathbf{u} \cdot \mathbf{n},
$$

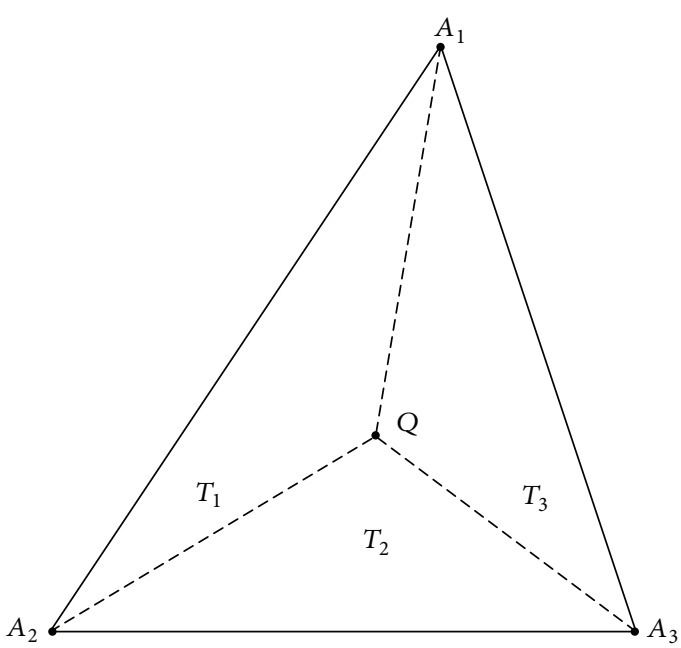

FIgURE 1: Element $T \in \mathscr{T}_{h}^{*}$ for a triangular mesh.

where $\mathbf{n}$ is the outward unit normal. We do not require either of the quantities $[p]$ or $\{\mathbf{u}\}$ on boundary edges, and we leave them undefined.

Multiplying the first and second equations in system (2) by $\mathbf{v} \in V_{h}$ and $p \in H_{h}$, respectively, and using the integration by parts formula in the equation, we have

$$
\begin{aligned}
& \sum_{T \in \mathscr{T}_{h}^{*}} \int_{T} M \mathbf{w} \cdot \mathbf{v} d x+\sum_{T \in \mathscr{T}_{h}^{*}} \int_{\partial T} q \mathbf{v} \cdot \mathbf{n} d s=0, \quad \forall \mathbf{v} \in V_{h}, \\
& \sum_{K \in \mathscr{T}_{h}} \int_{K} q_{t} p d x+\sum_{K \in \mathscr{T}_{h}} \int_{K} \nabla \cdot \mathbf{w} p d x \\
& \quad+\sum_{K \in \mathscr{T}_{h}} \int_{K} \int_{0}^{t} \nabla \cdot(D \mathbf{w}) p d \tau d x=(f, p), \quad \forall p \in H_{h},
\end{aligned}
$$

where $\mathbf{n}$ is the outward normal vector on $\partial T$. Let $T_{j} \in$ $\mathscr{T}_{h}^{*}(j=1,2,3)$ be the triangles in $K \in \mathscr{T}_{h}$. Then we have

$$
\begin{aligned}
\sum_{T \in \mathscr{T}_{h}^{*}} \int_{\partial T} q \mathbf{v} \cdot \mathbf{n} d s= & \sum_{K \in \mathscr{T}_{h}} \sum_{j=1}^{3} \int_{A_{j+1} \mathrm{QA}} q \mathbf{v} \cdot \mathbf{n} d s \\
& +\sum_{K \in \mathscr{T}_{h}} \int_{\partial K} q \mathbf{v} \cdot \mathbf{n} d s, \quad \forall \mathbf{v} \in V_{h},
\end{aligned}
$$

where $A_{4}=A_{1}$. A straightforward computation gives

$$
\begin{aligned}
& \sum_{K \in \mathscr{T}_{h}} \int_{\partial K} q \mathbf{v} \cdot \mathbf{n} d s \\
& \quad=\sum_{e \in \Gamma^{0}} \int_{e}[q] \cdot\{\mathbf{v}\} d s+\sum_{e \in \Gamma} \int_{e}\{q\} \cdot[\mathbf{v}] d s, \quad \forall \mathbf{v} \in V_{h} .
\end{aligned}
$$


Let $\int_{\Gamma} q d s=\sum_{e \in \Gamma} \int_{e} q d s$. Using the above formula and the fact that $[q]=0$ for $q \in H^{1}(\Omega)$ on $\Gamma^{0}$, (9) becomes

$$
\begin{aligned}
& \sum_{T \in \mathscr{T}_{h}^{*}} \int_{\partial T} q \mathbf{v} \cdot \mathbf{n} d s \\
& \quad=\sum_{K \in \mathscr{T}_{h}} \sum_{j=1}^{3} \int_{A_{j+1} \mathrm{Q} A_{j}} q \mathbf{v} \cdot \mathbf{n} d s+\sum_{e \in \Gamma} \int_{e}\{q\}[\mathbf{v}] d s, \quad \forall \mathbf{v} \in V_{h} .
\end{aligned}
$$

Then, system (8) can be rewritten as in the following:

$$
\begin{aligned}
& \sum_{T \in \mathscr{T}_{h}^{*}} \int_{T} M \mathbf{w} \cdot \mathbf{v} d x+\sum_{K \in \mathscr{T}_{h}} \sum_{j=1}^{3} \int_{A_{j+1} Q A_{j}} q \mathbf{v} \cdot \mathbf{n} d s \\
& \quad+\sum_{e \in \Gamma} \int_{e}\{q\}[\mathbf{v}] d s=0, \quad \forall \mathbf{v} \in V_{h}, \\
& \sum_{K \in \mathscr{T}_{h}} \int_{K} q_{t} p d x+\sum_{K \in \mathscr{T}_{h}} \int_{K} \nabla \cdot \mathbf{w} p d x \\
& \quad+\sum_{K \in \mathscr{T}_{h}} \int_{K} \int_{0}^{t} \nabla \cdot(D \mathbf{w}) p d \tau d x=(f, p), \quad \forall p \in H_{h} .
\end{aligned}
$$

$$
V_{h} \text { as }
$$

$$
\text { Let } U(h)=U_{h}+H^{2}(\Omega)^{2} \text {. Define a mapping } \gamma: U(h) \rightarrow
$$

$$
\left.\gamma \mathbf{u}\right|_{T}=\left.\frac{1}{h_{e}} \int_{e} \mathbf{u}\right|_{T} d s, \quad T \in \mathscr{T}_{h}^{*}
$$

where $h_{e}$ is the length of the edge $e$. For $\mathbf{u}=\left(u_{1}, u_{2}\right) \in U(h)$, $\gamma u_{i}(i=1,2)$ is defined as

$$
\left.\gamma u_{i}\right|_{T}=\left.\frac{1}{h_{e}} \int_{e} u_{i}\right|_{T} d s, \quad T \in \mathscr{T}_{h}^{*},(i=1,2) .
$$

Then the system (12) is equivalent to

$$
\begin{aligned}
& \sum_{T \in \mathscr{T}_{h}^{*}} \int_{T} M \mathbf{w} \cdot \gamma \mathbf{v} d x+\sum_{K \in \mathscr{T}_{h}} \sum_{j=1}^{3} \int_{A_{j+1} \mathrm{QA}} q \gamma \mathbf{v} \cdot \mathbf{n} d s \\
& \quad+\sum_{e \in \Gamma} \int_{e}\{q\}[\gamma \mathbf{v}] d s=0, \quad \forall \mathbf{v} \in U(h), \\
& \sum_{K \in \mathscr{T}_{h}} \int_{K} q_{t} p d x+\sum_{K \in \mathscr{T}_{h}} \int_{K} \nabla \cdot \mathbf{w} p d x \\
& \quad+\sum_{K \in \mathscr{T}_{h}} \int_{K} \int_{0}^{t} \nabla \cdot(D \mathbf{w}) p d \tau d x=(f, p), \quad \forall p \in H_{h} .
\end{aligned}
$$

Let

$$
\begin{aligned}
a_{0}(\mathbf{u}, \mathbf{v}) & :=\sum_{T \in \mathscr{T}_{h}^{*}} \int_{T} M \mathbf{u} \cdot \gamma \mathbf{v} d x, \\
b(\mathbf{v}, p) & :=\sum_{K \in \mathscr{T}_{h}} \sum_{j=1}^{3} \int_{A_{j+1} \mathrm{Q} A_{j}} p \gamma \mathbf{v} \cdot \mathbf{n} d s+\sum_{e \in \Gamma} \int_{e}\{p\}[\gamma \mathbf{v}] d s, \\
\mathcal{c}_{0}(\mathbf{u}, p) & :=\sum_{K \in \mathscr{T}_{h}} \int_{K} \nabla \cdot \mathbf{u} p d x, \\
\widetilde{\mathcal{C}}_{0}(\mathbf{u}, p) & :=\sum_{K \in \mathscr{T}_{h}} \int_{K} \nabla \cdot(D \mathbf{u}) p d x .
\end{aligned}
$$

Using the above bilinear forms, it is clear that system (15) can be rewritten as in the following:

$$
\begin{gathered}
a_{0}(\mathbf{w}, \mathbf{v})+b(\mathbf{v}, q)=0, \quad \forall \mathbf{v} \in U(h), \\
\left(q_{t}, p\right)+c_{0}(\mathbf{w}, p)+\int_{0}^{t} \widetilde{\mathcal{c}}_{0}(\mathbf{w}, p) d \tau=(f, p), \quad \forall p \in H_{h} .
\end{gathered}
$$

In order to define our numerical schemes, we introduce the bilinear forms as follows:

$$
\begin{aligned}
& a(\mathbf{u}, \mathbf{v}):=a_{0}(\mathbf{u}, \mathbf{v})+\alpha \sum_{e \in \Gamma} \frac{1}{h_{e}} \int_{e} M[\mathbf{u}][\mathbf{v}] d s \\
& c(\mathbf{u}, p):=c_{0}(\mathbf{u}, p)-\int_{\Gamma}\{p\}[\gamma \mathbf{u}] d s \\
& \tilde{c}(\mathbf{u}, p):=\widetilde{c}_{0}(\mathbf{u}, p)-\int_{\Gamma}\{p\}[\gamma \mathbf{u}] d s,
\end{aligned}
$$

where $\alpha>0$ is a parameter to be determined later. For the exact solution $(\mathbf{w}, q)$ of system (2), we have

$$
\begin{array}{ll}
a_{0}(\mathbf{w}, \mathbf{u})=a(\mathbf{w}, \mathbf{u}), & \forall \mathbf{u} \in U_{h}, \\
c_{0}(\mathbf{w}, p)=c(\mathbf{w}, p), & \forall p \in H_{h}, \\
\widetilde{c}_{0}(\mathbf{w}, p)=\widetilde{c}(\mathbf{w}, p), & \forall p \in H_{h} .
\end{array}
$$

Therefore, it follows from (17) that

$$
\begin{gathered}
a(\mathbf{w}, \mathbf{v})+b(\mathbf{v}, q)=0, \quad \forall \mathbf{v} \in U_{h}, \\
\left(q_{t}, p\right)+c(\mathbf{w}, p)+\int_{0}^{t} \widetilde{c}(\mathbf{w}, p) d \tau=(f, p), \quad \forall p \in H_{h} .
\end{gathered}
$$

The discontinuous mixed covolume scheme for (2) reads as follows. Seek $\left(\mathbf{w}_{h}, q_{h}\right) \in U_{h} \times H_{h}$ such that

$$
\begin{gathered}
a\left(\mathbf{w}_{h}, \mathbf{v}\right)+b\left(\mathbf{v}, q_{h}\right)=0, \quad \forall \mathbf{v} \in U_{h}, \\
\left(q_{h t}, p\right)+c\left(\mathbf{w}_{h}, p\right)+\int_{0}^{t} \widetilde{c}\left(\mathbf{w}_{h}, p\right) d \tau=(f, p), \quad \forall p \in H_{h},
\end{gathered}
$$


where $q_{h}(0)=\widetilde{q}_{h}(0), \mathbf{w}_{h}(0)=\widetilde{\mathbf{w}}_{h}(0), \widetilde{q}_{h}(0), \widetilde{\mathbf{w}}_{h}(0)$ will be given in Section 4.

Let $N>0$ be a positive integer; let $0=t^{0}<t^{1}<\cdots<t^{j}<$ $\cdots<t^{N}=T$ be a subdivision of time. $t^{j}=j \Delta t(0 \leq j \leq$ $N), \Delta t=T / N$. We use the backward Euler difference quotient

$$
\partial_{t} q_{h}^{j}=\frac{q_{h}^{j}-q_{h}^{j-1}}{\Delta t} \quad(j=1,2, \ldots, N)
$$

to approximate the differential quotient $\partial q_{h}^{j} / \partial t(j=1,2, \ldots$, $N)$ and the numerical integration $\Delta t \sum_{k=0}^{j-1} \nabla \cdot\left(D^{k} \mathbf{w}_{h}^{k}\right)$ to approximate the integration $\int_{0}^{t_{j}} \nabla \cdot\left(D \mathbf{w}_{h}\right) d \tau(j=1,2, \ldots, N)$ in the semidiscrete scheme; then we obtain the backward Euler fully discrete discontinuous mixed covolume scheme for the problem (1): find $\left(\mathbf{w}_{h}^{j}, q_{h}^{j}\right) \in U_{h} \times H_{h}(j=1,2, \ldots, N)$, such that

$$
\begin{gathered}
a\left(\mathbf{w}_{h}^{j}, \mathbf{v}\right)+b\left(\mathbf{v}, q_{h}^{j}\right)=0, \quad \forall \mathbf{v} \in U_{h}, \\
\left(\partial_{t} q_{h}^{j}, p\right)+c\left(\mathbf{w}_{h}^{j}, p\right)+\widetilde{c}\left(\Delta t \sum_{k=0}^{j-1} \mathbf{w}_{h}^{k}, p\right)=(f, p), \\
\forall p \in H_{h}, \quad j=1,2, \ldots, N,
\end{gathered}
$$

where $q_{h}^{0}=\widetilde{q}_{h}(0), \mathbf{w}_{h}^{0}=\widetilde{\mathbf{w}}_{h}(0), \widetilde{q}_{h}(0), \widetilde{\mathbf{w}}_{h}(0)$ will be given in Section 4 .

We define the following norms for $\mathbf{u} \in U(h)$ :

$$
\begin{gathered}
\mid\|\mathbf{u}\|\left\|_{\text {div }}^{2}=\right\| \mathbf{u}\left\|^{2}+\right\| \nabla_{h} \cdot \mathbf{u} \|^{2}+\sum_{e \in \Gamma} \frac{1}{h_{e}} \int_{e}[\mathbf{u}]^{2} d s, \\
|\|\mathbf{u}\||_{1}^{2}=\|\mathbf{u}\|^{2}+|\mathbf{u}|_{1, h}^{2}+\sum_{e \in \Gamma} \frac{1}{h_{e}} \int_{e}[\mathbf{u}]^{2} d s, \\
|\|\mathbf{u}\||^{2}=|\|\mathbf{u}\||_{1}^{2}+\sum_{K \in \mathscr{T}_{h}} h_{K}^{2}|\mathbf{u}|_{2, K}^{2},
\end{gathered}
$$

where $\nabla_{h} \cdot \mathbf{u}$ is the function whose restriction to each element $K \in \mathscr{T}_{h}$ is equal to $\nabla \cdot \mathbf{u}$ and $|\mathbf{u}|_{1, h}^{2}=\sum_{K \in \mathscr{T}_{h}}|\mathbf{u}|_{1, K}^{2}$.

We will introduce some useful Lemmas; for more details, see [6].

Lemma 1. For $\mathbf{u}, \mathbf{v} \in U(h)$, we have

$$
a(\mathbf{u}, \mathbf{v}) \leq C|\|\mathbf{u}\||_{\text {div }}|\|\mathbf{v}\||_{\text {div }} .
$$

Lemma 2. For $(\mathbf{u}, p) \in U(h) \times L^{2}(\Omega)$, we have

$$
b(\mathbf{u}, p)=-c(\mathbf{u}, p) .
$$

Lemma 3. For $(\mathbf{u}, p) \in U(h) \times L^{2}(\Omega)$, we have

$$
b(\mathbf{u}, p) \leq C|\|\mathbf{u}\||\left(\|p\|+\left(\sum_{K \in \mathscr{T}_{h}} h_{K}^{2}|p|_{1, K}^{2}\right)^{1 / 2}\right) ;
$$

if $(\mathbf{u}, p) \in U_{h} \times H_{h}$, then

$$
\begin{aligned}
& b(\mathbf{u}, p) \leq C|\|\mathbf{u}\|| \cdot\|p\|, \\
& \tilde{c}(\mathbf{u}, p) \leq C|\|\mathbf{u}\|| \cdot\|p\| .
\end{aligned}
$$

Lemma 4. Let $Z_{h}=\left\{\mathbf{u} \mid \mathbf{u} \in U_{h}, c(\mathbf{u}, p)=0, \forall p \in H_{h}\right\}$; for any $\mathbf{u} \in Z_{h}$, there is a constant $C_{0}$ independent of $h$ such that, for $\alpha$ is large enough,

$$
a(\mathbf{u}, \mathbf{u}) \geq C_{0}|\|\mathbf{u}\||_{\text {div }}^{2} .
$$

\section{Existence and Uniqueness for Discontinuous Mixed Covolume Approximations}

In this section, we prove the discontinuous mixed covolume formulation has a unique solution in the finite element space $U_{h} \times H_{h}$.

Theorem 5. The semidiscrete discontinuous mixed covolume scheme (21) has a unique solution in the space $U_{h} \times H_{h}$.

Proof. Only prove that homogenous equation

$$
\begin{gathered}
a\left(\mathbf{w}_{h}, \mathbf{u}\right)+b\left(\mathbf{u}, q_{h}\right)=0, \quad \forall \mathbf{u} \in U_{h}, \\
\left(q_{h t}, p\right)+c\left(\mathbf{w}_{h}, p\right)+\int_{0}^{t} \widetilde{\mathcal{c}}_{0}\left(\mathbf{w}_{h}, p\right)=0, \quad \forall p \in H_{h}, \\
q_{h}(0)=0, \quad \mathbf{w}_{h}(0)=0,
\end{gathered}
$$

of (21) exists unique zero solution since the number of unknowns is the same as number of line equations.

By letting $\mathbf{v}=\mathbf{w}_{h}$ in the first formula of (30) and $p=q_{h}$ in the second formula of (30), using Lemma 2 , the sum of (30) gives

$$
a\left(\mathbf{w}_{h}, \mathbf{w}_{h}\right)+\left(q_{h t}, q_{h}\right)=-\int_{0}^{t} \widetilde{\mathcal{c}}_{0}\left(\mathbf{w}_{h}, q_{h}\right) .
$$

Using $(1 / 2)(d / d t)\left(q_{h}, q_{h}\right)=\left(q_{h t}, q_{h}\right)$ and Lemmas 3 and 4 , we have that

$$
\begin{aligned}
& C_{0} \mid\left\|\mathbf{w}_{h}\right\|_{\text {div }}^{2}+\frac{1}{2} \frac{d}{d t}\left\|q_{h}\right\|^{2} \\
& \quad \leq C \int_{0}^{t}\left|\left\|\mathbf{w}_{h}\right\|\right|\left\|q_{h}\right\| d \tau \leq C \int_{0}^{t}\left|\left\|\mathbf{w}_{h}\right\|\right|_{\text {div }}\left\|q_{h}\right\| d \tau .
\end{aligned}
$$

Using Hölder inequality and Gronwall Lemma, we get

$$
C_{0}\left\|\mathbf{w}_{h}\right\|_{\text {div }}^{2}+\frac{1}{2} \frac{d}{d t}\left\|q_{h}\right\|^{2} \leq C \int_{0}^{t}\left\|q_{h}\right\|^{2} d \tau .
$$

Integrating the above formula, we get

$$
\begin{aligned}
& 2 C_{0} \int_{0}^{t} \mid\left\|\mathbf{w}_{h}\right\|_{\text {div }}^{2} d \tau+\left\|q_{h}\right\|^{2} \\
& \quad \leq C \int_{0}^{t} \int_{0}^{s}\left\|q_{h}\right\|^{2} d \tau d s \leq C \int_{0}^{t}\left\|q_{h}\right\|^{2} d \tau .
\end{aligned}
$$

Then $\left\|q_{h}\right\|=0,\left\|\left|\mathbf{w}_{h} \|\right|_{\text {div }}=0\right.$. So $q_{h}=0, \mathbf{w}_{h}=\mathbf{0}, t \in(0, T]$. This completes the proof.

Theorem 6. The fully discrete discontinuous mixed covolume method defined in (23) has a unique solution in the finite element space $U_{h} \times H_{h}$ if $\Delta t$ is sufficiently small. 
Proof. Only prove that homogenous equation

$$
\begin{gathered}
a\left(\mathbf{w}_{h}^{j}, \mathbf{v}\right)+b\left(\mathbf{v}, q_{h}^{j}\right)=0, \quad \forall \mathbf{v} \in U_{h}, \\
\left(\partial_{t} q_{h}^{j}, p\right)+c\left(\mathbf{w}_{h}^{j}, p\right)+\widetilde{c}\left(\Delta t \sum_{k=0}^{j-1} \mathbf{w}_{h}^{k}, p\right)=0, \\
\forall p \in H_{h}, \quad j=1,2, \ldots, N,
\end{gathered}
$$

of (23) exists unique zero solution since the number of unknowns is the same as number of line equations.

By letting $\mathbf{v}=\mathbf{w}_{h}^{j}$ in the first formula of (35) and $p=q_{h}^{j}$ in the second formula of (35), using Lemma 2, the sum of (35) gives

$$
\begin{array}{r}
a\left(\mathbf{w}_{h}^{j}, \mathbf{w}_{h}^{j}\right)+\left(\partial_{t} q_{h}^{j}, q_{h}^{j}\right)=-\widetilde{c}\left(\Delta t \sum_{k=0}^{j-1} \mathbf{w}_{h}^{k}, q_{h}^{j}\right), \\
j=1,2, \ldots, N .
\end{array}
$$

Using Lemmas 3 and 4 and

$$
\begin{aligned}
& \left(\partial_{t} q_{h}^{j}, q_{h}^{j}\right) \\
& \quad=\frac{1}{\Delta t}\left(q_{h}^{j}-q_{h}^{j-1}, q_{h}^{j}\right) \\
& \quad=\frac{1}{2 \Delta t}\left[\left(q_{h}^{j}, q_{h}^{j}\right)-\left(q_{h}^{j-1}, q_{h}^{j-1}\right)+\left(q_{h}^{j}-q_{h}^{j-1}, q_{h}^{j}-q_{h}^{j-1}\right)\right] \\
& \quad=\frac{1}{2 \Delta t}\left[\left\|q_{h}^{j}\right\|^{2}-\left\|q_{h}^{j-1}\right\|^{2}+\left\|q_{h}^{j}-q_{h}^{j-1}\right\|^{2}\right] \\
& \quad \geq \frac{1}{2 \Delta t}\left[\left\|q_{h}^{j}\right\|^{2}-\left\|q_{h}^{j-1}\right\|^{2}\right]
\end{aligned}
$$

we have from (36) that

$$
\begin{aligned}
& 2 C_{0} \Delta t\left|\left\|\mathbf{w}_{h}^{j}\right\|\right|_{\text {div }}^{2}+\left\|q_{h}^{j}\right\|^{2}-\left\|q_{h}^{j-1}\right\|^{2} \\
& \leq C \Delta t\left[(\Delta t)^{2} \mid\left\|\sum_{k=0}^{j-1} \mathbf{w}_{h}^{k}\right\|\left\|_{\text {div }}^{2}+\right\| q_{h}^{j} \|^{2}\right], \quad j=1,2, \ldots, N .
\end{aligned}
$$

Adding the above inequality with $j$ from 1 to $i$, using $q_{h}^{0}=0$ and the discrete Gronwall inequality, when $\Delta t$ is sufficiently small, we have

$$
2 C_{0} \Delta t \sum_{j=1}^{i}\left|\left\|\mathbf{w}_{h}^{j}\right\|\right|_{\text {div }}^{2}+\left\|q_{h}^{i}\right\|^{2} \leq 0 \quad(i=1,2, \ldots, N) .
$$

Hence we have $\left\|q_{h}^{i}\right\|^{2}=0$ and $\left|\left\|\mathbf{w}_{h}^{i}\right\|\right|_{\text {div }}^{2}=0(i=1,2, \ldots, N)$; that is, $q_{h}^{i}=0$ and $\mathbf{w}_{h}^{i}=\mathbf{0}(i=1,2, \ldots, N)$. This completes the proof.

\section{A Discontinuous Mixed Covolume Elliptic Projection}

Define an operator $\pi_{K}$ from $H^{1}(K)$ to $P_{1}(K)$ by requiring that, for any $\forall u \in H^{1}(K)$,

$$
\int_{e_{i}} \pi_{K} u d s=\int_{e_{i}} u d s, \quad(i=1,2,3)
$$

where $e_{i}(i=1,2,3)$ are the three sides of the element $K \in$ $\mathscr{T}_{h}$. It was proved in [5] that

$$
\left|\pi_{K} u-u\right|_{s, K} \leq h^{2-s}|u|_{2, K}, \quad \forall u \in H^{2}(K),(s=0,1,2) .
$$

For any $\mathbf{u} \in H_{0}^{1}(\Omega)^{2}$, define $\Pi_{1} \mathbf{u} \in U_{h}$ by

$$
\left.\left(\Pi_{1} \mathbf{u}\right)_{i}\right|_{K}=\Pi_{K} u_{i}, \quad \forall K \in \mathscr{T}_{h}, \quad(i=1,2) .
$$

Using the definition of $\Pi_{1}$ and integration by parts, we can show that

$$
c\left(\mathbf{u}-\Pi_{1} \mathbf{u}, p\right)=0, \quad \forall p \in H_{h} .
$$

It was proved in [6] that

$$
\left|\left\|\mathbf{w}-\Pi_{1} \mathbf{w}\right\|\right|_{\text {div }} \leq \operatorname{ch}\|\mathbf{w}\|_{2} .
$$

Let $\Pi_{2}$ be the projection from $L_{0}^{2}(\Omega)$ to the finite element space $H_{h}$.

Define a discontinuous mixed covolume elliptic projection by requiring that, finding $\widetilde{\mathbf{w}}_{h}, \widetilde{q}_{n}:(0, t) \rightarrow U_{h} \times H_{h}$, such that

$$
\begin{gathered}
a\left(\mathbf{w}-\widetilde{\mathbf{w}}_{h}, \mathbf{v}\right)+b\left(\mathbf{v}, q-\widetilde{q}_{h}\right)=0, \quad \forall \mathbf{v} \in U_{h}, \\
c\left(\mathbf{w}-\widetilde{\mathbf{w}}_{h}, p\right)=0, \quad \forall p \in H_{h} .
\end{gathered}
$$

It was proved in [15] that the above formula has a unique solution and the error estimates in the following Theorem 7.

Theorem 7. Let $\left(\widetilde{\mathbf{w}}_{h}, \widetilde{q}_{h}\right) \in U_{h} \times H_{h}$ be the solution of (45) and $(\mathbf{w}, q) \in H^{2}(\Omega)^{2} \times H^{1}(\Omega)$ the solution of $(20)$. Then there exists a positive constant $C$ independent of h such that

$$
\left.\left\|\mathbf{w}-\widetilde{\mathbf{w}}_{h}\right\|\right|_{\text {div }}+\left\|q-\tilde{q}_{h}\right\| \leq C h\left(\|\mathbf{w}\|_{2}+\|q\|_{1}\right) .
$$

Theorem 8. Let $\left(\widetilde{\mathbf{w}}_{h}, \widetilde{q}_{h}\right) \in U_{h} \times H_{h}$ be the solution of (45) and $(\mathbf{w}, q) \in H^{2}(\Omega)^{2} \times H^{1}(\Omega)$ the solution of $(20)$. Then there exists a positive constant $C$ independent of $h$ such that

$$
\begin{aligned}
& \left|\left\|\left(\mathbf{w}-\widetilde{\mathbf{w}}_{h}\right)_{t}\right\|\right|_{\text {div }}+\left\|\left(q-\widetilde{q}_{h}\right)_{t}\right\| \\
& \quad \leq \operatorname{Ch}\left(\left\|\mathbf{w}_{t}\right\|_{2}+\left\|q_{t}\right\|_{1}+\|\mathbf{w}\|_{2}+\|q\|_{1}\right) .
\end{aligned}
$$

Differentiating each equation of (45) on $t$ and using (43), (44) we can prove this theorem in the same way as [15]. 


\section{Error Estimates for Semidiscrete Method}

In this section, we will establish the error estimates in the $H$ (div) and $L^{2}$ norms for the semidiscrete discontinuous mixed covolume method.

Theorem 9. Let $\left(\mathbf{w}_{h}, q_{h}\right) \in U_{h} \times H_{h}$ be the solution of (21) and $q_{h}(0)=\widetilde{q}_{h}(0),(\mathbf{w}, q) \in H^{2}(\Omega)^{2} \times H^{1}(\Omega)$ the solution of $(2)$. Then there exists a positive constant $C$ independent of $h$ such that

$$
\begin{aligned}
& \left.\quad\left\|\mathbf{w}-\mathbf{w}_{h}\right\|\right|_{\text {div }}+\left\|q-q_{h}\right\| \\
& \leq \operatorname{Ch}\left[\int_{0}^{t}\left(\left\|\mathbf{w}_{t}\right\|_{2}+\left\|q_{t}\right\|_{1}+\|\mathbf{w}\|_{2}+\|q\|_{1}\right) d \tau+\|\mathbf{w}\|_{2}+\|q\|_{1}\right] .
\end{aligned}
$$

Proof. Let $\xi=\widetilde{q}_{h}-q_{h}, \eta=\widetilde{\mathbf{w}}_{h}-\mathbf{w}_{h}$. Subtracting the two equations of (21) from those of (20), respectively, we have

$$
\begin{gathered}
a\left(\mathbf{w}-\mathbf{w}_{h}, \mathbf{v}\right)+b\left(\mathbf{v}, q-q_{h}\right)=0, \quad \forall \mathbf{v} \in U_{h}, \\
\left(q_{t}-q_{h_{t}}, p\right)+c\left(\mathbf{w}-\mathbf{w}_{h}, p\right)+\int_{0}^{t} \widetilde{c}\left(\mathbf{w}-\mathbf{w}_{h}, p\right) d \tau=0, \\
\forall p \in H_{h} .
\end{gathered}
$$

Using (45), we have

$$
\begin{gathered}
a(\eta, \mathbf{v})+b(\mathbf{v}, \xi)=0, \quad \forall \mathbf{v} \in U_{h}, \\
\left(q_{t}-q_{h_{t}}, p\right)+c(\eta, p) \\
=-\int_{0}^{t} \widetilde{c}(\eta, p) d \tau-\int_{0}^{t} \widetilde{c}\left(\mathbf{w}-\widetilde{\mathbf{w}}_{h}, p\right) d \tau, \quad \forall p \in H_{h} .
\end{gathered}
$$

Differentiating the first equation of (50) on $t$, we have that

$$
a\left(\eta_{t}, \mathbf{v}\right)+b\left(\mathbf{v}, \xi_{t}\right)=0, \quad \forall \mathbf{v} \in U_{h} .
$$

By letting $p=\xi_{t}$ in the second formula of (50) and letting $\mathbf{v}=\eta$ in (51), using Lemma 2 , the sum of them gives

$$
\begin{aligned}
& a\left(\eta_{t}, \eta\right)+\left(\xi_{t}, \xi_{t}\right) \\
& \quad=\left(\left(\tilde{p}_{h}-p\right)_{t}, \xi_{t}\right)-\int_{0}^{t} \tilde{c}\left(\eta, \xi_{t}\right) d \tau-\int_{0}^{t} \tilde{c}\left(\mathbf{w}-\widetilde{\mathbf{w}}_{h}, \xi_{t}\right) d \tau .
\end{aligned}
$$

Using

$$
a\left(\eta_{t}, \eta\right)=\frac{1}{2} \frac{d}{d t} a(\eta, \eta)-\frac{1}{2}\left(M_{t} \eta, \eta\right)
$$

and Lemmas 1 and 3 gives

$$
\begin{aligned}
\frac{1}{2} \frac{d}{d t} a(\eta, \eta) & +\left\|\xi_{t}\right\|^{2} \\
\leq C & {\left[\left\|\xi_{t}\right\| \cdot\left\|\left(\tilde{p}_{h}-p\right)_{t}\right\|+\mid\|\eta\|_{\text {div }}^{2}\right.} \\
& \left.+\int_{0}^{t}|\|\eta\||_{\text {div }}\left\|\xi_{t}\right\| d \tau+\int_{0}^{t}\left|\left\|\mathbf{w}-\widetilde{\mathbf{w}}_{h}\right\|\right|_{\text {div }}\left\|\xi_{t}\right\| d \tau\right] .
\end{aligned}
$$

Multiplying the equation above with 2, integrating them from 0 to $t$ and using Hölder inequality, $\epsilon$-inequality, Gronwall inequality, Lemma 4, and (47), we can get

$$
\begin{aligned}
\|\| \eta \|_{\text {div }}^{2} & +\frac{1}{C_{0}}\left(\int_{0}^{t}\left\|\xi_{t}\right\| d \tau\right)^{2} \\
& \leq \operatorname{Ch}^{2}\left(\int_{0}^{t}\left(\left\|\mathbf{w}_{t}\right\|_{2}+\left\|q_{t}\right\|_{1}+\|\mathbf{w}\|_{2}+\|q\|_{1}\right) d \tau\right)^{2}
\end{aligned}
$$

so

$$
\begin{gathered}
\mid\|\eta\|_{\text {div }} \leq C h \int_{0}^{t}\left(\left\|\mathbf{w}_{t}\right\|_{2}+\left\|q_{t}\right\|_{1}+\|\mathbf{w}\|_{2}+\|q\|_{1}\right) d \tau, \\
\int_{0}^{t}\left\|\xi_{t}\right\| d \tau \leq C h \int_{0}^{t}\left(\left\|\mathbf{w}_{t}\right\|_{2}+\left\|q_{t}\right\|_{1}+\|\mathbf{w}\|_{2}+\|q\|_{1}\right) d \tau ;
\end{gathered}
$$

hence

$$
\|\xi\| \leq \int_{0}^{t}\left\|\xi_{t}\right\| d \tau \leq C h \int_{0}^{t}\left(\left\|\mathbf{w}_{t}\right\|_{2}+\left\|q_{t}\right\|_{1}+\|\mathbf{w}\|_{2}+\|q\|_{1}\right) d \tau .
$$

Now, using the triangle inequality, (46), (56), and (58), we get

$$
\begin{aligned}
& \left|\left\|\mathbf{w}-\mathbf{w}_{h}\right\|\right|_{\text {div }}+\left\|q-q_{h}\right\| \\
& \leq C h\left[\int_{0}^{t}\left(\left\|\mathbf{w}_{t}\right\|_{2}+\left\|q_{t}\right\|_{1}+\|\mathbf{w}\|_{2}+\|q\|_{1}\right) d \tau+\|\mathbf{w}\|_{2}+\|q\|_{1}\right] .
\end{aligned}
$$

The proof is complete.

\section{Error Estimates for Fully Discrete Method}

Let $\xi^{j}=\widetilde{q}_{h}^{j}-q_{h}^{j}, \zeta^{j}=\widetilde{q}_{h}^{j}-q^{j}, \eta^{j}=\widetilde{\mathbf{w}}_{h}^{j}-\mathbf{w}_{h}^{j}(j=0,1, \ldots$, $N)$, and then the error estimates for the backward Euler fully discrete discontinuous mixed covolume method in the $H$ (div) and $L^{2}$ norms are provided in next two theorems.

Theorem 10. Let $(\mathbf{w}, q) \in H^{2}(\Omega)^{2} \times H^{1}(\Omega)$ be the solution of (2) and $\left(\mathbf{w}_{h}^{j}, q_{h}^{j}\right) \in U_{h} \times H_{h}(j=1,2, \ldots, N)$ the solution of (23) with $t=t_{j}(j=1,2, \ldots, N)$, respectively. If $q_{h}^{0}=\tilde{q}_{h}(0)=$ $q_{0}, \mathbf{w}_{h}^{0}=\widetilde{\mathbf{w}}_{h}(0)=\mathbf{w}_{0}$, then there exists a positive constant $C$ independent of $h$ and $\Delta t$ such that

$$
\begin{gathered}
\max _{0 \leq i \leq N}\left\|q^{i}-q_{h}^{i}\right\|+\max _{0 \leq i \leq N}\left|\left\|\mathbf{w}^{i}-\mathbf{w}_{h}^{i}\right\|\right|_{\text {div }} \\
\leq C \Delta t\left(\left\|q_{t t}\right\|_{L^{\infty}\left(L^{2}\right)}+\left\|\mathbf{w}_{t}\right\|_{L^{\infty}\left(H^{2}\right)}\right) \\
+C h\left(\left\|\mathbf{w}_{t}\right\|_{L^{\infty}\left(H^{2}\right)}+\left\|q_{t}\right\|_{L^{\infty}\left(H^{1}\right)}\right. \\
\left.+\|\mathbf{w}\|_{L^{\infty}\left(H^{2}\right)}+\|q\|_{L^{\infty}\left(H^{1}\right)}\right) .
\end{gathered}
$$


Journal of Applied Mathematics

7

Proof. Subtracting the two equations of (23) from (20), respectively, with $t=t^{j}(j=0,1, \ldots, N)$, we can get the error equation:

$$
\begin{gathered}
a\left(\mathbf{w}^{j}-\mathbf{w}_{h}^{j}, \mathbf{v}\right)+b\left(\mathbf{v}, q^{j}-q_{h}^{j}\right)=0, \quad \forall \mathbf{v} \in U_{h}, \\
\left(q_{t}^{j}-\partial_{t} q_{h}^{j}, p\right)+c\left(\mathbf{w}^{j}-\mathbf{w}_{h}^{j}, p\right)+\int_{0}^{t^{j}} \widetilde{c}(\mathbf{w}, p) d \tau \\
-\widetilde{c}\left(\Delta t \sum_{k=0}^{j-1} \mathbf{w}_{h}^{k}, p\right)=0, \quad \forall p \in H_{h}, j=1,2, \ldots, N .
\end{gathered}
$$

Choosing $\mathbf{v}=\eta^{j}(j=1,2, \ldots, N)$ and $p=\xi^{j}(j=$ $1,2, \ldots, N)$ in the two equations of (61), adding them together, and using Lemma 2 , discontinuous mixed covolume elliptic projection with $t=t^{j}(j=0,1, \ldots, N)$, we have

$$
\begin{aligned}
& a\left(\eta^{j}, \eta^{j}\right)+\left(\partial_{t} \xi^{j}, \xi^{j}\right) \\
& =\left(\partial_{t} q^{j}-q_{t}^{j}, \xi^{j}\right)+\left(\partial_{t} \zeta^{j}, \xi^{j}\right)-\int_{0}^{t^{j}} \widetilde{c}\left(\mathbf{w}-\widetilde{\mathbf{w}}_{h}, \xi^{j}\right) d \tau \\
& \quad-\widetilde{c}\left(\Delta t \sum_{k=0}^{j-1} \eta^{k}, \xi^{j}\right)-\int_{0}^{t^{j}} \widetilde{c}\left(\widetilde{\mathbf{w}}_{h}, \xi^{j}\right) d \tau+\widetilde{c}\left(\Delta t \sum_{k=0}^{j-1} \widetilde{\mathbf{w}}_{h}^{k}, \xi^{j}\right) .
\end{aligned}
$$

First, we estimate the left item of (62). Using Lemma 4, we have

$$
a\left(\eta^{j}, \eta^{j}\right) \geq C_{0} \mid\left\|\eta^{j}\right\|_{\text {div }}^{2}
$$

$$
\begin{aligned}
& \left(\partial_{t} \xi^{j}, \xi^{j}\right) \\
& \quad=\frac{1}{\Delta t}\left(\xi^{j}-\xi^{j-1}, \xi^{j}\right) \\
& \quad=\frac{1}{2 \Delta t}\left[\left(\xi^{j}, \xi^{j}\right)-\left(\xi^{j-1}, \xi^{j-1}\right)+\left(\xi^{j}-\xi^{j-1}, \xi^{j}-\xi^{j-1}\right)\right] \\
& \quad=\frac{1}{2 \Delta t}\left[\left\|\xi^{j}\right\|^{2}-\left\|\xi^{j-1}\right\|^{2}+\left\|\xi^{j}-\xi^{j-1}\right\|^{2}\right] \\
& \quad>\frac{1}{2 \Delta t}\left[\left\|\xi^{j}\right\|^{2}-\left\|\xi^{j-1}\right\|^{2}\right] .
\end{aligned}
$$

Then, we estimate the right item of (62). From

$$
\begin{aligned}
\| \partial_{t} q^{j} & -q_{t}^{j} \|^{2} \\
& =\left\|\frac{1}{\Delta t} \int_{t^{j-1}}^{t^{j}}\left(t^{j-1}-t\right) q_{t t} d t\right\|^{2}
\end{aligned}
$$

$$
\begin{aligned}
& \leq \int_{\Omega}\left(\frac{1}{\Delta t} \int_{t^{j-1}}^{t^{j}}\left(t^{j-1}-t\right) q_{t t} d t\right)^{2} d x \\
& \leq \frac{1}{(\Delta t)^{2}} \int_{\Omega}\left(\int_{t^{j-1}}^{t^{j}}\left(t^{j-1}-t\right)^{2} d t \int_{t^{j-1}}^{t^{j}} q_{t t}^{2} d t\right) d x \\
& \leq C(\Delta t)^{2}\left\|q_{t t}\right\|_{L^{\infty}\left(L^{2}\right)}^{2}
\end{aligned}
$$

we have

$$
\left(\partial_{t} q^{j}-q_{t}^{j}, \xi^{j}\right) \leq C(\Delta t)\left\|q_{t t}\right\|_{L^{\infty}\left(L^{2}\right)}\left\|\xi^{j}\right\|
$$

$\left\|\partial_{t} \zeta^{j}\right\|$

$$
\begin{aligned}
& =\left\|\frac{\zeta^{j}-\zeta^{j-1}}{\Delta t}\right\|=\left\|\frac{1}{\Delta t} \int_{t^{j-1}}^{t^{j}} \zeta_{t} d t\right\| \\
& \leq \frac{1}{\Delta t} \int_{t^{j-1}}^{t^{j}}\left\|\zeta_{t}\right\| d t \\
& \leq \frac{C h}{\Delta t} \int_{t^{j-1}}^{t^{j}}\left(\left\|\mathbf{w}_{t}\right\|_{2}+\left\|q_{t}\right\|_{1}+\|\mathbf{w}\|_{2}+\|q\|_{1}\right) d t \\
& \leq C h\left(\left\|\mathbf{w}_{t}\right\|_{L^{\infty}\left(H^{2}\right)}+\left\|q_{t}\right\|_{L^{\infty}\left(H^{1}\right)}+\|\mathbf{w}\|_{L^{\infty}\left(H^{2}\right)}+\|q\|_{L^{\infty}\left(H^{1}\right)}\right),
\end{aligned}
$$

and therefore

$$
\begin{aligned}
& \left(\partial_{t} \zeta^{j}, \xi^{j}\right) \\
& \leq \quad \operatorname{Ch}\left(\left\|\mathbf{w}_{t}\right\|_{L^{\infty}\left(H^{2}\right)}+\left\|q_{t}\right\|_{L^{\infty}\left(H^{1}\right)}+\|\mathbf{w}\|_{L^{\infty}\left(H^{2}\right)}+\|q\|_{L^{\infty}\left(H^{1}\right)}\right) \\
& \quad \times\left\|\xi^{j}\right\| .
\end{aligned}
$$

Using Lemma 3, we can get

$$
\begin{gathered}
-\int_{0}^{t^{j}} \widetilde{c}\left(\mathbf{w}-\widetilde{\mathbf{w}}_{h}, \xi^{j}\right) d \tau \\
\leq C \int_{0}^{t^{j}} \mid\left\|\mathbf{w}-\widetilde{\mathbf{w}}_{h}\right\|_{\text {div }} d \tau\left\|\xi^{j}\right\| \\
\leq C h\left(\|\mathbf{w}\|_{L^{\infty}\left(H^{2}\right)}+\|p\|_{L^{\infty}\left(H^{1}\right)}\right)\left\|\xi^{j}\right\|, \\
-\widetilde{c}\left(\Delta t \sum_{k=0}^{j-1} \eta^{k}, \xi^{j}\right) \leq C(\Delta t) \sum_{k=0}^{j-1}\left|\left\|\eta^{k}\right\|\right|_{\text {div }}\left\|\xi^{j}\right\|, \\
-\int_{0}^{t^{j}} \widetilde{c}\left(\widetilde{\mathbf{w}}_{h}, \xi^{j}\right) d \tau+\widetilde{c}\left(\Delta t \sum_{k=0}^{j-1} \widetilde{\mathbf{w}}_{h}^{k}, \xi^{j}\right) \\
=\widetilde{c}\left(\sum_{k=0}^{j-1} \int_{t^{k}}^{t^{k+1}}\left(\widetilde{\mathbf{w}}_{h}^{k}-\widetilde{\mathbf{w}}_{h}(\tau)\right) d \tau, \xi^{j}\right)
\end{gathered}
$$




$$
\begin{aligned}
& \leq C\left\|\sum_{k=0}^{j-1} \int_{t^{k}}^{t^{k+1}} \int_{t^{k}}^{\tau} \widetilde{\mathbf{w}}_{h_{t}} d s d \tau\right\|\left\|\xi^{j}\right\| \\
& \leq C(\Delta t)\left\|\mathbf{w}_{t}\right\|_{L^{\infty}\left(H^{2}\right)}\left\|\xi^{j}\right\| .
\end{aligned}
$$

Substituting the estimations above into (62), multiplying them with $\Delta t$, adding them with $j$ from 1 to $i$, and using $\xi^{0}=$ 0 , we have

$$
\begin{aligned}
& \left\|\xi^{i}\right\|^{2}+2 C_{0} \Delta t \sum_{j=1}^{i} \mid\left\|\eta^{j}\right\|_{\text {div }}^{2} \\
& \leq C(\Delta t)^{2}\left(\left\|q_{t t}\right\|_{L^{\infty}\left(L^{2}\right)}+\left\|\mathbf{w}_{t}\right\|_{L^{\infty}\left(H^{2}\right)}\right)^{2} \\
& \quad+C(\Delta t)^{2} \sum_{k=0}^{j-1} \mid\left\|\eta^{k}\right\|_{\text {div }}^{2}+C \Delta t \sum_{j=1}^{i}\left\|\xi^{j}\right\|^{2} \\
& \quad+C h^{2}\left(\left\|\mathbf{w}_{t}\right\|_{L^{\infty}\left(H^{2}\right)}+\left\|q_{t}\right\|_{L^{\infty}\left(H^{1}\right)}+\|\mathbf{w}\|_{L^{\infty}\left(H^{2}\right)}+\|q\|_{L^{\infty}\left(H^{1}\right)}\right)^{2}
\end{aligned}
$$

Using the discrete Gronwall inequality, we have

$$
\begin{aligned}
& \max _{0 \leq i \leq N}\left\|\xi^{i}\right\|+\max _{0 \leq i \leq N} \mid\left\|\eta^{i}\right\| \|_{\text {div }} \\
& \leq C \Delta t\left(\left\|q_{t t}\right\|_{L^{\infty}\left(L^{2}\right)}+\left\|\mathbf{w}_{t}\right\|_{L^{\infty}\left(H^{2}\right)}\right) \\
& \quad+C h\left(\left\|\mathbf{w}_{t}\right\|_{L^{\infty}\left(H^{2}\right)}+\left\|q_{t}\right\|_{L^{\infty}\left(H^{1}\right)}+\|\mathbf{w}\|_{L^{\infty}\left(H^{2}\right)}+\|q\|_{L^{\infty}\left(H^{1}\right)}\right) .
\end{aligned}
$$

From the formula above and (46) and using the triangle inequality, we have

$$
\begin{aligned}
& \max _{0 \leq i \leq N}\left\|q^{i}-q_{h}^{i}\right\|+\max _{0 \leq i \leq N} \mid\left\|\mathbf{w}^{i}-\mathbf{w}_{h}^{i}\right\|_{\text {div }} \\
& \leq C \Delta t\left(\left\|q_{t t}\right\|_{L^{\infty}\left(L^{2}\right)}+\left\|\mathbf{w}_{t}\right\|_{L^{\infty}\left(H^{2}\right)}\right) \\
& \quad+C h\left(\left\|\mathbf{w}_{t}\right\|_{L^{\infty}\left(H^{2}\right)}+\left\|q_{t}\right\|_{L^{\infty}\left(H^{1}\right)}+\|\mathbf{w}\|_{L^{\infty}\left(H^{2}\right)}+\|q\|_{L^{\infty}\left(H^{1}\right)}\right) .
\end{aligned}
$$

This completes the proof.

\section{Conflict of Interests}

The author declares that there is no conflict of interests regarding the publication of this paper.

\section{Acknowledgments}

The author acknowledges a project supported by the fund of National Natural Science (11171193) and a project of Shandong Province Science and Technology Development Program (2012GGB01198).

\section{References}

[1] V. Thomée and N. Y. Zhang, "Error estimates for semidiscrete finite element methods for parabolic integro-differential equations," Mathematics of Computation, vol. 53, no. 187, pp. 121-139, 1989.

[2] A. K. Pani and G. Fairweather, " $H^{1}$-Galerkin mixed finite element methods for parabolic partial integro-differential equations," IMA Journal of Numerical Analysis, vol. 22, no. 2, pp. 231$252,2002$.

[3] C. Chen, V. Thomee, and L. B. Wahlbin, "Finite element approximation of a parabolic integro-differential equation with a weakly singular kernel," Mathematics of Computation, vol. 58, no. 198, pp. 587-602, 1992.

[4] A. Zhu and Q. Yang, "Expanded mixed finite element methods for parabolic integro-differential equations," Journal of Shandong Normal University, vol. 19, no. 2, pp. 10-14, 2004.

[5] A. Zhu, Z. Jiang, and Q. Xu, "Expanded mixed covolume method for a linear integro-differential equation of parabolic type," Numerical Mathematics, vol. 31, no. 3, pp. 193-205, 2009.

[6] W. H. Reed and T. R. Hill, "Triangular mesh methods for the neutron transport equation," Tech. Rep. LA-UR-73-479, Los Alamos Scientific Laboratory, Los Alamos, NM, USA, 1973.

[7] B. Cockburn, S. Hou, and C. W. Shu, "The Runge-Kutta local projection discontinuous Galerkin finite element method for conservation laws. IV. The multidimensional case," Mathematics of Computation, vol. 54, no. 190, pp. 545-581, 1990.

[8] B. Cockburn, G. E. Karniaddakis, and C. W. Shu, The Development of Discontinuous Galerkin Methods, Springer, Berlin, Germany, 2000.

[9] B. Cockburn and C. Shu, "The local discontinuous Galerkin method for time-dependent convection-diffusion systems," SIAM Journal on Numerical Analysis, vol. 35, no. 6, pp. 24402463, 1998.

[10] B. Riviere, M. F. Wheeler, and V. Girault, "Improved energy estimates for interior penalty, constrained and discontinuous Galerkin methods for elliptic problems-part I," Computational Geosciences, vol. 3, no. 3-4, pp. 337-360, 1999.

[11] D. N. Arnold, F. Brezzi, B. Cockburn, and L. D. Marini, "Unified analysis of discontinuous Galerkin methods for elliptic problems," SIAM Journal on Numerical Analysis, vol. 39, no. 5, pp. 1749-1779, 2002.

[12] X. Ye, "A new discontinuous finite volume method for elliptic problems," SIAM Journal on Numerical Analysis, vol. 42, no. 3, pp. 1062-1072, 2004.

[13] X. Ye, "A discontinuous finite volume method for the Stokes problems," SIAM Journal on Numerical Analysis, vol. 44, no. 1, pp. 183-198, 2006.

[14] C. Bi and J. Geng, "Discontinuous finite volume element method for parabolic problems," Numerical Methods for Partial Differential Equations, vol. 26, no. 2, pp. 367-383, 2010.

[15] Q. Yang and Z. Jiang, "A discontinuous mixed covolume method for elliptic problems," Journal of Computational and Applied Mathematics, vol. 235, no. 8, pp. 2467-2476, 2011.

[16] A. Zhu and Z. Jiang, "Discontinuous mixed covolume methods for parabolic problems," The Scientific World Journal, vol. 2014, Article ID 867863, 8 pages, 2014.

[17] R. A. Adams, Sobolev Spaces, Academic Press, New York, NY, USA, 1975. 


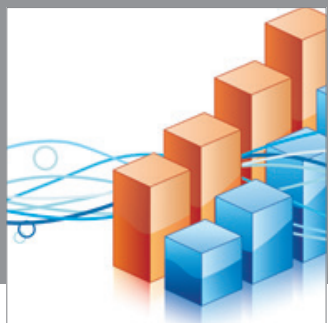

Advances in

Operations Research

mansans

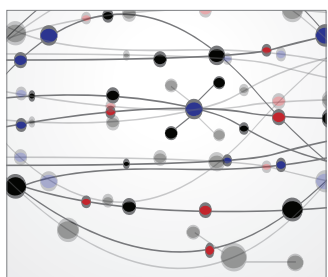

The Scientific World Journal
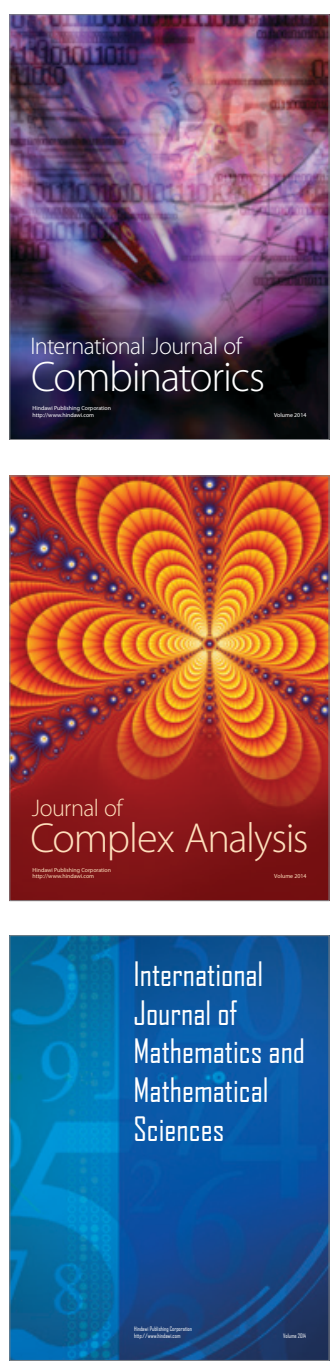
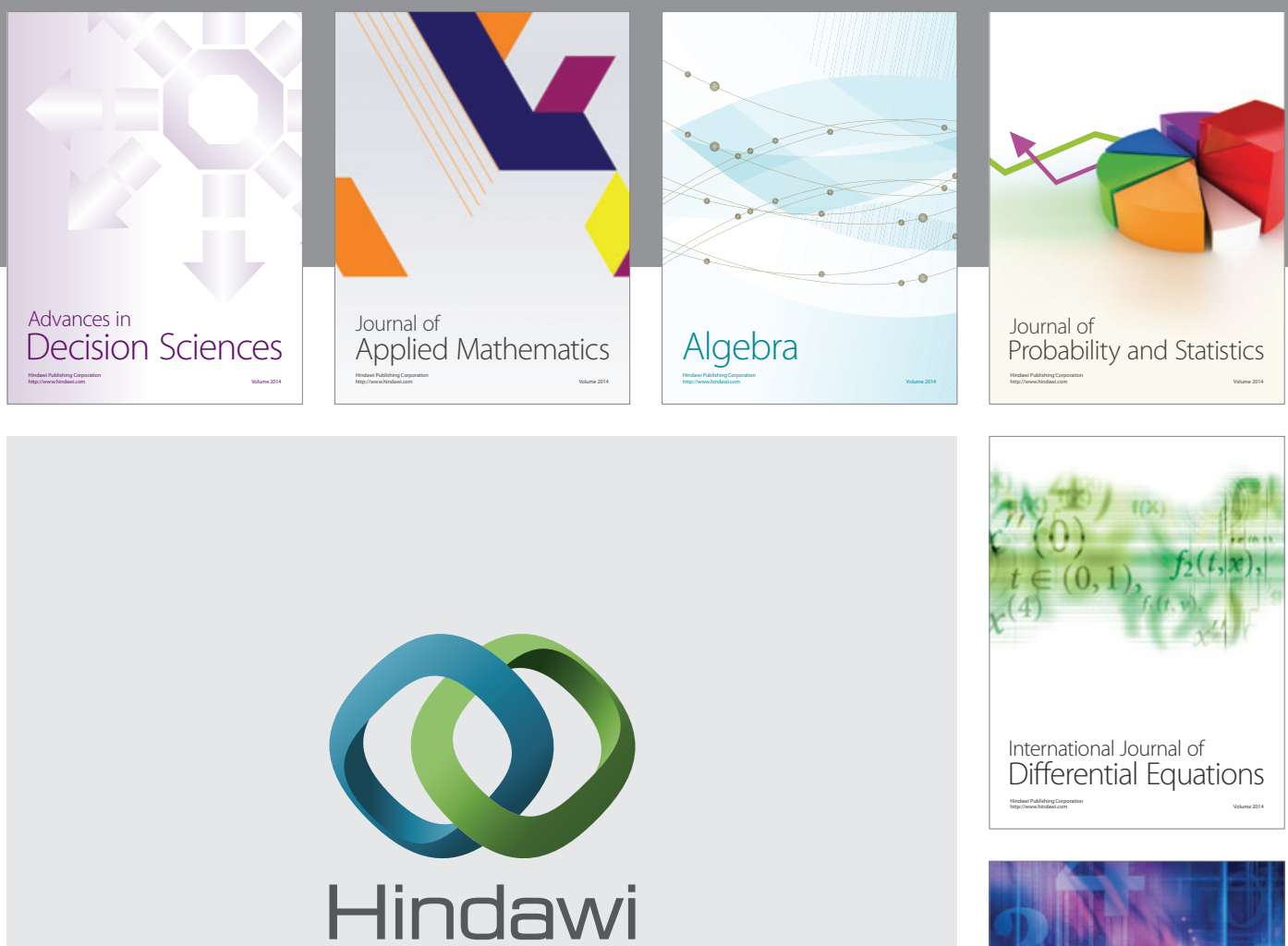

Submit your manuscripts at http://www.hindawi.com
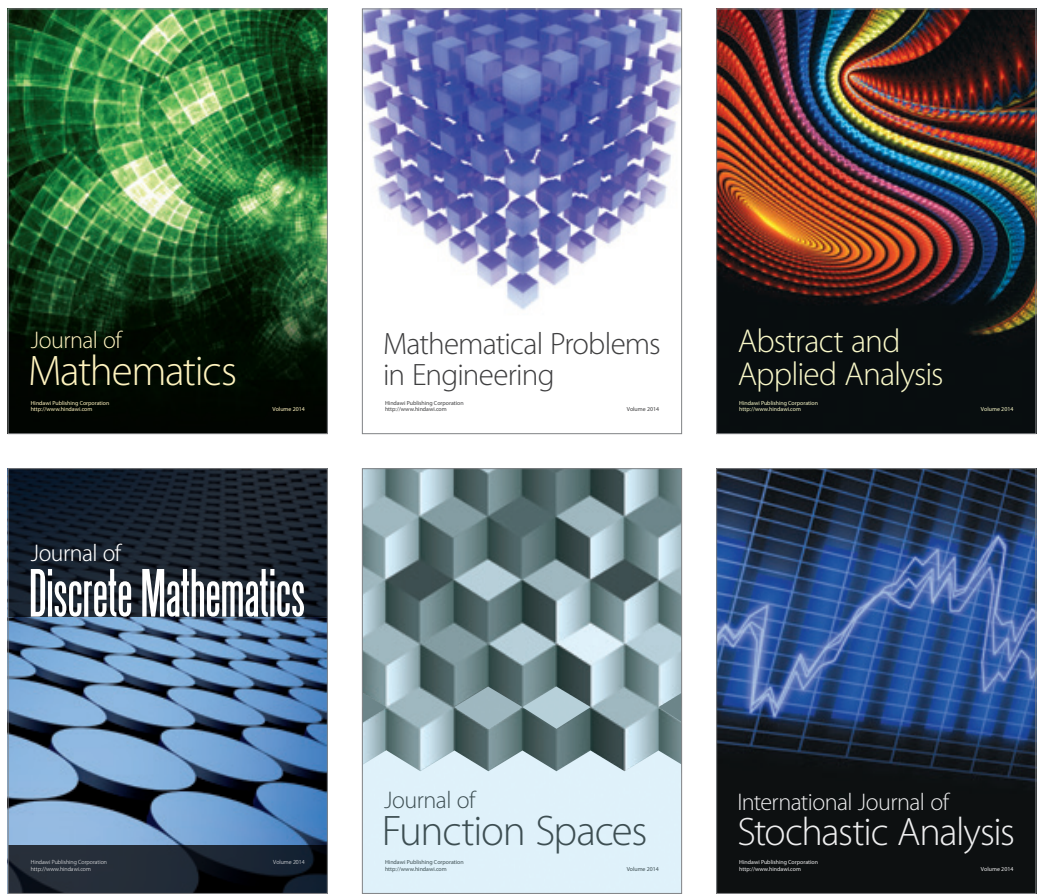

Journal of

Function Spaces

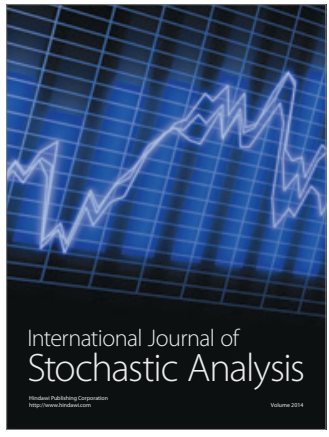

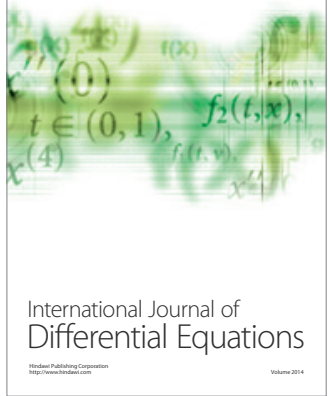
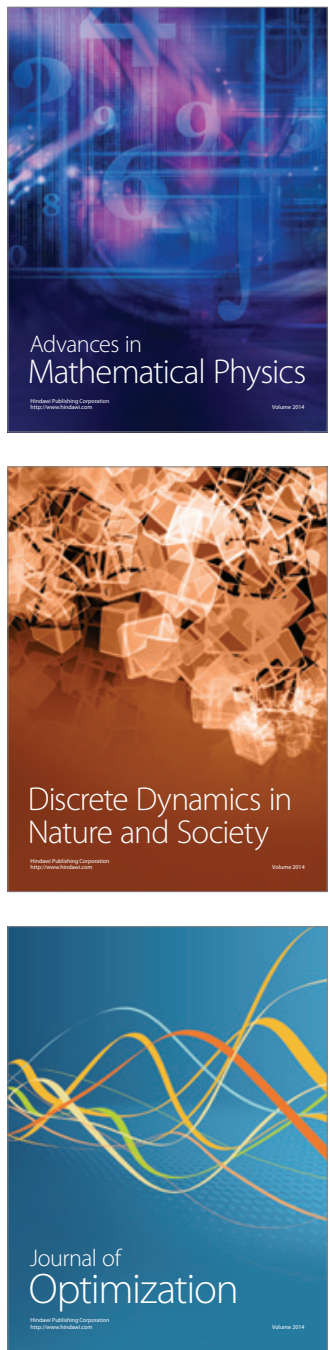